\title{
Perceptual learning transfer in an appetitive Pavlovian task
}

\author{
Antonio A. Artigas ${ }^{1} \cdot$ Jose Prados $^{2}$
}

Published online: 5 August 2016

(C) Psychonomic Society, Inc. 2016

\begin{abstract}
In two experiments, rats were given intermixed or blocked preexposure to two similar compound stimuli, AX and BX. Following preexposure, conditioning trials took place in which AX (Experiment 1) or a novel compound stimulus NX (Experiment 2) was paired with a food-unconditioned stimulus in an appetitive Pavlovian preparation. Animals that were given alternated preexposure showed lower generalization from AX to BX (Experiment 1) and from NX to a new compound, ZX (Experiment 2), than animals that were given blocked preexposure, a perceptual learning and a perceptual learning transfer effect, respectively.
\end{abstract}

Keywords Perceptual learning · Elemental learning · Configural learning $\cdot$ Stimulus sampling theory $\cdot$ Selective attention

A conditioned response developed to one conditioned stimulus (CS) is likely to generalize to a novel stimulus to the extent that they share some common elements. Any two stimuli can be conceptualized as $\mathrm{AX}$ and $\mathrm{BX}$, where $\mathrm{A}$ and $\mathrm{B}$ are the unique elements that would help discrimination, and $\mathrm{X}$ correspond to the common elements that contribute to the ambiguity of the situation. To successfully discriminate between AX and BX, the animal would need to attend, for example, to the unique distinctive features and ignore the common elements.

Jose Prados

jpg19@le.ac.uk

1 Departament de Psicologia Bàsica and Institut de Recerca en Cervell, Conducta i Cognició (IR3C), Universitat de Barcelona,

Barcelona, Spain

2 Department of Neuroscience Psychology and Behaviour, University of Leicester, Lancaster Road, Leicester LE1 9HN, UK
Interestingly, learning processes are engaged that direct the attention of the animals to the unique elements and away from the common elements in situations in which the animals are simply exposed to the compound stimuli in the absence of any reward. We refer to these learning processes as perceptual learning (e.g., Gibson, 1969; Hall, 2003; McLaren \& Mackintosh, 2000; see Mitchell \& Hall, 2014, for a full review).

Perceptual learning processes are more likely to affect stimuli that are presented intermixed (AX, BX, AX, BX) than stimuli presented in separate blocks of trials (AX, AX . . . BX, BX). This intermixed-blocked effect, an instance of perceptual learning, has been widely researched in a variety of species and procedures: in domestic chicks using visual discriminations (Honey, Bateson, \& Horn, 1994); in humans using visual (Lavis \& Mitchell, 2006) and flavor (Dwyer, Hodder, \& Honey, 2004) discriminations; and in rats using flavor (e.g., Artigas, Sansa, \& Prados, 2006; Blair \& Hall, 2003; Prados, Hall, \& Leonard, 2004), spatial (e.g., Prados, Artigas, \& Sansa, 2007), and acoustic discriminations (Mondragon \& Murphy, 2010). Mondragon and Murphy (2010), for example, using a standard appetitive Pavlovian preparation, exposed two groups of rats to AX and BX according to an intermixed or a blocked schedule. Following conditioning with the compound AX, animals were tested in the presence of the compound $\mathrm{BX}$ - a generalization test. Animals given intermixed preexposure showed better discrimination between AX and BX than did animals in the blocked condition. The experiment by Mondragon and Murphy (2010) remains the only instance of perceptual learning reported using a standard appetitive Pavlovian conditioning technique in rats.

Enhanced discrimination between AX and BX could be the consequence of the action of a number of processes. On the one hand, modern theories of perceptual learning suggest that intermixed, but not blocked, preexposure increases the salience of the unique features $\mathrm{A}$ and $\mathrm{B}$; these theories tend to 
assume no differences in the salience of the common element $\mathrm{X}$ in the two preexposure schedules (e.g., Hall, 2003). However, earlier theories of perceptual learning suggested a second mechanism that might affect the discriminability of $\mathrm{AX}$ and $\mathrm{BX}$ - namely, the reduced attention paid to the irrelevant common feature, X (e.g., Gibson, 1969).

There is evidence that intermixed preexposure tends to maintain high salience of A and B compared to the blocked preexposure procedure (e.g., Artigas, Sansa, Blair, Hall, \& Prados, 2006; Blair \& Hall, 2003; Contel, Sansa, Artigas, \& Prados, 2011; Hall, Blair, \& Artigas, 2006). Furthermore, Mondragon and Murphy (2010; see also Mondragon \& Hall, 2002), have reported evidence that support the notion that the common element $\mathrm{X}$ is less salient after intermixed than after blocked preexposure. Using a standard appetitive conditioning task, Mondragon and Murphy observed that animals that were given intermixed preexposure to $\mathrm{AX}$ and $\mathrm{BX}$ showed less conditioning to the common element $\mathrm{X}$ than those given blocked preexposure. According to standard associative learning theory (e.g., Mackintosh, 1975; Pearce \& Hall, 1980; Rescorla \& Wagner, 1972), a low in salience X element would be less likely to associate with the unconditioned stimulus (US) during the conditioning phase of a typical perceptual learning experiment (in which the AX compound is paired with the US), reducing mediated generalization to the BX element during the generalization test. Also, lack of attention to the common element $\mathrm{X}$ would also enhance the discrimination between similar compounds (as anticipated by Gibson, 1969) in experiments in which the generalization test is not used. For example, studies in which human participants were required to discriminate between different versions of the same photograph of a face created with a morphing procedure showed that preexposure to the common element $\mathrm{X}$ alone could enhance discrimination between compound stimuli with novel unique characteristics (AX and BX). However, alternated preexposure to the two compound stimuli, $\mathrm{AX}$ and $\mathrm{BX}$, was found to be a more effective procedure to enhance their discriminability (e.g., Mundy, Honey, \& Dwyer 2007). These results suggest the existence of two mechanisms contributing to the perceptual learning effect - one based upon the increased salience or effectiveness of A and B and another based upon the reduced salience of $X$.

Increased salience of A and B in the intermixed schedule would result in enhanced discriminability of the preexposed compound stimuli AX and BX exclusively. However, assuming the existence of a second mechanism, reduced salience of $\mathrm{X}$ in the intermixed procedure, would extend the facilitatory effect of preexposure to similar compound stimuli containing new unique features (like NX and ZX, for example). Artigas and Prados (2014) recently reported some evidence for such a perceptual learning transfer effect using a flavor discrimination task. After receiving intermixed or blocked preexposure to $\mathrm{AX}$ and $\mathrm{BX}$, rats were given conditioning trials with a novel compound stimulus, NX. It was expected that a less salient $\mathrm{X}$ element in the intermixed group should acquire less control over the conditioned response during conditioning with NX than in the blocked group, reducing the generalization (mediated by the common element) to novel compound stimuli containing the $\mathrm{X}$ element. In agreement with this hypothesis, animals that were given intermixed preexposure to $\mathrm{AX}$ and $\mathrm{BX}$ showed lower generalization of the aversive response conditioned to NX to a new compound, ZX, than animals that were given blocked preexposure.

As pointed out previously, the Mondragon and Murphy (2010) study reports the only instance of a perceptual learning effect using a standard appetitive Pavlovian conditioning task. The aim of Experiment 1 was to obtain a double replica of this result by using a set of stimuli that were intended to be identical to those used by Mondragon and Murphy, as well as with a new set of stimuli. These two sets of stimuli were then used to explore the perceptual learning transfer effect reported by Artigas and Prados (2014) in a flavor aversion procedure using a more orthodox appetitive procedure.

\section{Experiment 1}

Mondragon and Murphy (2010) suggested that one reason for the lack of evidence of perceptual learning with standard Pavlovian procedures could be that perceptual learning might be evident only when the stimuli are initially rather difficult to discriminate. In previous unsuccessful attempts (e.g., Honey, 1990), animals were required to discriminate between relatively distinctive stimuli - tones and clicks - arguably an easy task. Preexposure has been shown to benefit subsequent discrimination only when the to-be-discriminated stimuli share a high proportion of common elements. It has often been shown, for example, that adding explicit common features facilitates discrimination after intermixed (but not blocked) preexposure (e.g., Mackintosh, Kaye, \& Bennett, 1991; Prados, Chamizo, \& Mackintosh, 1999). In their experiments, Mondragon and Murphy (2010) observed perceptual learning in a relatively difficult task that made use of two similar compound stimuli formed by two pure tones (which differed in their frequency; the unique features $\mathrm{A}$ and $\mathrm{B}$ ) and a white noise delivered through an additional speaker (the explicit common element X).

In Experiment 1 we sought to replicate this experiment using exactly the same stimuli used by Mondragon and Murphy (Experiment 1A). In Experiment 1B we aimed to replicate the effect using two additional compound stimuli in which the elements A and B were two discontinued tones presented at different rates of intermittence. The reason why we wanted to replicate the effect with a new set of stimuli is our interest in the perceptual learning transfer effect, which is the objective of Experiment 2: To assess the transfer of perceptual learning, we would need two sets of stimuli (AX and 
$\mathrm{BX}$; NX and ZX) that can be reliably used to demonstrate the perceptual learning effect.

In both Experiments $1 \mathrm{~A}$ and $1 \mathrm{~B}$, animals in the group intermixed were given presentations of $\mathrm{AX}$ and $\mathrm{BX}$ in an alternated schedule (AX, BX, AX, BX ...), whereas animals in the group blocked were exposed to the same stimuli in two separate blocks of identical trials (AX, AX ... BX, BX). All the animals were then given conditioning trials in which the AX compound was paired with the presentation of a food unconditioned stimulus (US). Differences in discrimination were assessed by comparing responding during a generalization test to $\mathrm{BX}$ (see experimental designs in Table 1). If intermixed preexposure is more effective in engaging perceptual learning processes that increase the discriminability of the $\mathrm{AX}$ and $\mathrm{BX}$ compounds, then less responding to the BX compound could be expected after intermixed than blocked preexposure.

\section{Method}

\section{Subjects}

The subjects of Experiment 1A were 16 experimentally naïve male hooded Long Evans rats (ad lib body weight range $345: 437 \mathrm{~g}$ ); the subjects of Experiment $1 \mathrm{~B}$ were 16 naïve male hooded Long Evans rats (ad lib body weight range 329:438 g). They were housed, in standard transparent plastic cages, $24 \times$ $50 \times 14.5 \mathrm{~cm}$, in groups of two or three animals, in a colony room that was artificially lit from 9:00 a.m. to 9:00 p.m. daily, with free access to water. The animals were handled, weighed, and fed a restricted amount of food at the end of each session to keep them at $85 \%$ of their ad lib body weight for the course of the experiment.

\section{Apparatus}

Four identical Modular Operant Box chambers $(25.0 \mathrm{~cm} \times 25.0 \mathrm{~cm} \times 25.0 \mathrm{~cm})$ from Panlab (Model LE1005, Panlab/Harvard Apparatus) were used. The chambers were inserted in sound- and light-attenuating boxes (Model LE26,

Table 1 Experimental designs

\begin{tabular}{lllll}
\hline & Group & Preexposure & Conditioning & Test \\
\hline Experiment 1 & Intermixed & $\mathrm{AX} / \mathrm{BX}$ & $\mathrm{AX}+$ & $\mathrm{BX}$ \\
& Blocked & $\mathrm{AX}-\mathrm{BX}$ & $\mathrm{AX}+$ & $\mathrm{BX}$ \\
Experiment 2 & Intermixed & $\mathrm{AX} / \mathrm{BX}$ & $\mathrm{NX}+$ & $\mathrm{ZX}$ \\
& Blocked & $\mathrm{AX}-\mathrm{BX}$ & $\mathrm{NX}+$ & $\mathrm{ZX}$ \\
\hline
\end{tabular}

A, B, N, Z, and X represent different acoustic stimuli. Stimuli separated by a forward slash (/) in the preexposure phase were presented on alternate trials; stimuli separated by a dash $(-)$ were presented in separate blocks of trials. + represents the delivery of a food-US (two food pellets)
Panlab/Harvard Apparatus), with background noise produced by ventilation fans ( $\approx 70 \mathrm{~dB})$. Each chamber was dimly illuminated by a shielded houselight operating at $20 \mathrm{~V}$ located on the wall opposite to the food tray. The floor of each chamber consisted of 20 tubular stainless steel bars; these bars were perpendicular to the wall where the food tray was located (Model LE100501, Panlab/Harvard Apparatus). This wall and the opposite one were made of aluminum; the ceiling and remaining walls were of clear methacrylate. A magazine pellet dispenser (Model LE100550, Panlab/Harvard Apparatus) delivered 45-mg Dustless Precision Pellets (Bio Serv; Rodent Purified Diet) into the food tray. A head entry into the food tray was recorded by interruption of a LED photocell (Model LE105-51, Panlab/Harvard Apparatus). Two speakers (Model LE100543, Panlab/Harvard Apparatus) were attached to the top sides of the front wall. The left speaker could deliver four different 15 -s tones. In Experiment $1 \mathrm{~A}$, a $3.2 \mathrm{kHz}(80 \mathrm{~dB})$ and a $9.5 \mathrm{kHz}(80 \mathrm{~dB})$ tone were used as the elements $\mathrm{A}$ and $\mathrm{B}$ of the experimental design. In Experiment $1 \mathrm{~B}$, two $5 \mathrm{kHz}(80 \mathrm{~dB})$ discontinuous tones that could be presented at two different rates of intermittency were used as the elements $\mathrm{A}$ and $\mathrm{B}$ of the experimental design. One of the tones was presented at a low rate of intermittency ( $200 \mathrm{~ms}$ of the tone alternated with $200 \mathrm{~ms}$ of silence), whereas the other was presented at a high rate of intermittency $(200 / 100 \mathrm{~ms})$. The right speaker delivered a $75 \mathrm{~dB}$ white noise, the $\mathrm{X}$ element of the experimental design, in both experiments. A computer running the Packwin software platform 2.0 for Windows XP controlled experimental events.

\section{Procedure}

There were three phases in the experiment: preexposure, conditioning, and test. Throughout the experiment, rats were presented with trials separated by a variable ITI with a mean of 240 s. During the first 2 days of the experiment, animals were exposed to the compound stimuli $\mathrm{AX}$ and $\mathrm{BX}$ (five presentations of each compound every day). The initial order (counterbalanced) in which the stimuli were exposed in Day 1 was reversed on Day 2, and the identity of the first stimulus was also counterbalanced. In group intermixed the stimuli were exposed in an alternated fashion (e.g., AX, BX, AX, BX ....). In group blocked the stimuli were presented in separate blocks of identical trials (e.g., AX, AX . . BX, BX). Two sessions of conditioning followed, each of which comprised 10 presentations of AX followed by two pellets of food. During the final day of the experiment, the animals were given a test in which the compound BX was presented six times in extinction. The amount of time the animals kept their head in the food tray was recorded during the stimulus presentation (CS period) and during the $15 \mathrm{~s}$ that preceded it (the Pre-CS period). A difference score in which time responding during the Pre-CS was subtracted from that recorded during the CS was computed and used as a response measure. 


\section{Data analysis}

The analysis of this measure of performance was conducted with analyses of variance (ANOVA) using a rejection criterion of $p<.05$. The reported effect size for ANOVA with more than one factor is partial eta squared $\left(\eta_{\mathrm{p}}{ }^{2}\right)$, whereas for comparisons between two means it is eta squared $\left(\eta^{2}\right)$. For both measures of effect size, $95 \%$ confidence intervals (CIs) were computed using the method reported by Steiger (2004).

\section{Results and discussion}

Experiments $1 \mathrm{~A}$ and $1 \mathrm{~B}$ produced the same pattern of results. Figure 1 shows the group means for the magazine approach response calculated from difference scores (CS-Pre-CS) during the conditioning trials with the AX compound (in blocks of five conditioning trials) for the two replicas. ANOVAs with Preexposure (intermixed vs. blocked) and Blocks of Trials as factors showed a significant effect of Blocks of Trials for Experiment $1 \mathrm{~A}, F(3,42)=7.66, p=.00, \eta_{\mathrm{p}}{ }^{2}=.35,95 \% \mathrm{CI}$ $[.09, .50]$, and for Experiment $1 \mathrm{~B}, F(3,42)=4.64, p=.00, \eta_{\mathrm{p}}{ }^{2}$ $=.24,95 \% \mathrm{CI}[.02, .40]$. The remaining factors and interactions were all nonsignificant in both replicas, maximum $F(3$, 42) $=1.22$.

The same analyses were carried out on the Pre-CS levels of responding during the conditioning phase of Experiments $1 \mathrm{~A}$ and 1B. In Experiment 1A, the mean Pre-CS scores for the four blocks of conditioning trials were $1.67( \pm 0.66$ SEM $), 1.50$ $( \pm 0.59), 1.90( \pm 0.51)$, and $2.27( \pm 0.43)$ for group intermixed; $1.42( \pm 0.32), 1.80( \pm 0.36), 1.86( \pm 0.53)$ and $2.19( \pm 0.30)$ for group blocked. In Experiment 1B, the mean Pre-CS scores for the four blocks of conditioning trials were $1.37( \pm 0.39), 1.30$

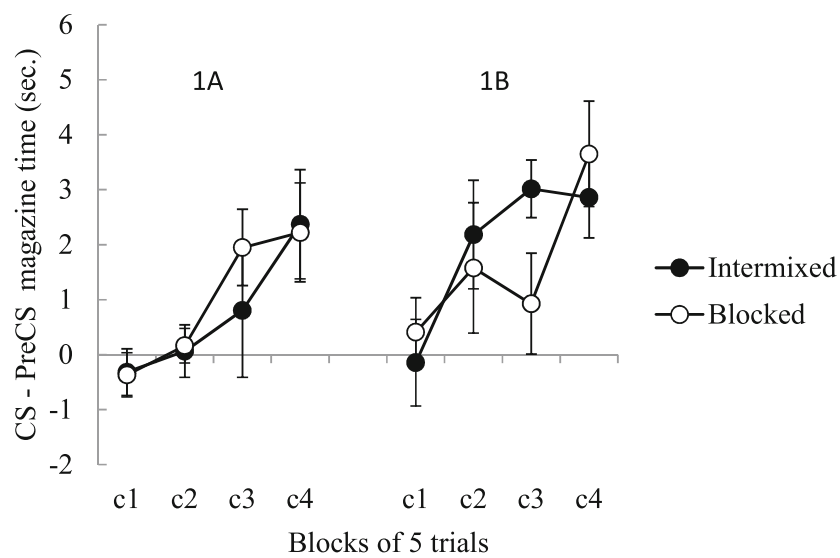

Fig. 1 Mean time $( \pm S E M)$ of magazine approach response calculated from difference scores (CS-Pre-CS) during the four blocks of conditioning trials with the compound $\mathrm{AX}$ for groups intermixed and blocked in Experiment 1 (Experiment 1A, left hand panel; Experiment $1 \mathrm{~B}$, right hand panel)
$( \pm 0.66), 2.03( \pm 0.65)$, and $1.59( \pm 0.56)$ for group intermixed; $1.43( \pm 0.34), 1.84( \pm 0.63), 1.72( \pm 0.48)$, and $1.86( \pm 0.48)$ for group blocked. ANOVAs with Preexposure (intermixed vs. blocked) and Blocks of Trials as factors showed no significant effects, $F \mathrm{~s}<1$.

Figure 2 shows the group means for the magazine approach response during three blocks of two test trials with the $\mathrm{BX}$ compound for experiments $1 \mathrm{~A}$ and $1 \mathrm{~B}$. ANOVAs with Preexposure (intermixed vs. blocked) and Blocks of Test Trials as the factors showed a significant effect of Preexposure for Experiment $1 \mathrm{~A}, F(1,14)=5.03, p=.04$, $\eta_{\mathrm{p}}{ }^{2}=.26,95 \%$ CI $[.00, .54]$, and for Experiment $1 \mathrm{~B}, F(1$, $14)=5.30, p=.03, \eta_{\mathrm{p}}{ }^{2}=.27,95 \% \mathrm{CI}[.00, .55]$. The factor Blocks of Test Trials was also significant in Experiment 1B, $F(3,42)=4.29, p=.02, \eta_{\mathrm{p}}{ }^{2}=.23,95 \%$ CI $[.00, .39]$. The remaining main factors and interactions were all nonsignificant in both replicas, maximum $F(3,42)=1.36$.

The same analyses were carried out on the Pre-CS scores during the test phase of Experiments $1 \mathrm{~A}$ and 1B. In Experiment $1 \mathrm{~A}$, the mean Pre-CS scores for the three blocks of test trials were $1.68( \pm 0.66), 2.18( \pm 1.03)$, and $1.98( \pm 1.05)$ for group intermixed; $1.30( \pm 0.63), 2.40( \pm 0.75)$, and $1.39( \pm 0.93)$ for group blocked. In Experiment 1B, the mean Pre-CS scores for the three blocks of conditioning trials were $0.63( \pm 0.63), 1.11$ $( \pm 0.52)$, and $1.22( \pm 0.43)$ for group intermixed; $0.82( \pm 0.34)$, $1.49( \pm 0.67)$, and $0.65( \pm 0.34)$ for group blocked. ANOVAs with Preexposure (intermixed vs. blocked) and Blocks of Test Trials as factors showed no significant effects, $F_{\mathrm{S}}<1$.

Experiment 1A fully replicates the perceptual learning effect observed by Mondragon and Murphy (2010) using a standard appetitive Pavlovian conditioning task: intermixed preexposure to $\mathrm{AX}$ and $\mathrm{BX}$ significantly reduced the generalization between these compound stimuli, comparison made with the blocked preexposure condition. Experiment $1 \mathrm{~B}$ stands as a second replica of the effect using an alternative set of stimuli (with discontinuous tones as the unique elements $\mathrm{A}$ and $\mathrm{B}$ ).

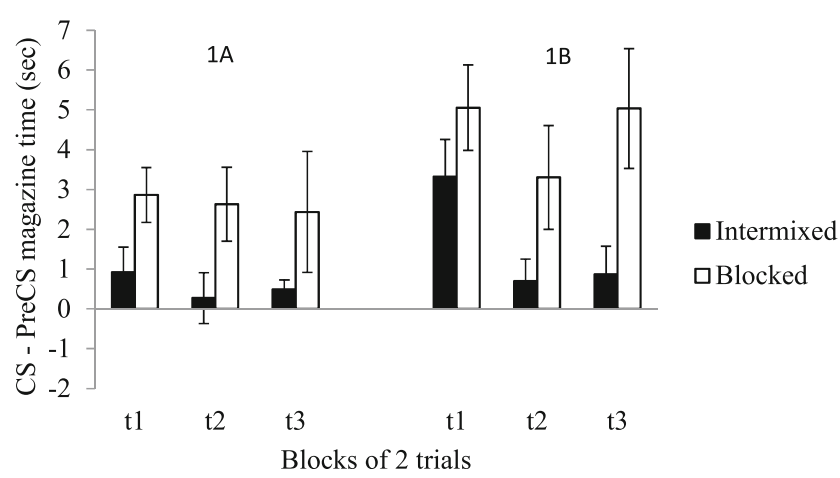

Fig. 2 Mean time $( \pm S E M)$ of magazine approach response calculated from difference scores (CS-Pre-CS) during the three blocks of test trials with the compound BX for groups intermixed and blocked in Experiment 1 (Experiment 1A, left hand panel; Experiment 1B, right hand panel) 
Most of current theories of perceptual learning assume preexposure to alter the perceptual characteristics of complex stimuli through intermixed preexposure by increasing, on the one hand, the perceptual effectiveness of the distinctive characteristics A and B (e.g., Hall, 2003; Honey \& Bateson, 1996; McLaren \& Mackintosh, 2000; Mitchell, Nash, \& Hall, 2008), and reducing, on the other hand, the effectiveness of the common elements, X (e.g., Gibson, 1969). Increased attention paid to the unique features A and B could perfectly well account for the intermixed-blocked effect observed in this experiment without having to assume any role for the common element $\mathrm{X}$.

Artigas and Prados (2014) hypothesized that a common element $\mathrm{X}$ with a reduced salience would acquire less predictive value during the conditioning phase with $\mathrm{AX}$, lowering the generalization (mediated by the common element) of the conditioned response to the BX element. Unfortunately, the contribution of the $\mathrm{X}$ element to the standard perceptual learning effect would be masked by the well-established contribution of the increased in salience unique elements A and B: On the one hand, A can be expected to acquire most of the predictive value during conditioning, overshadowing $X$; on the other hand, the presence of a salient B element would interfere with the retrieval of the conditioned response controlled by the $\mathrm{X}$ element at the time of the generalization test with BX (e.g., Artigas, Sansa, Blair, Hall, \& Prados, 2006).

To go round this problem and assess the contribution of the $\mathrm{X}$ element to the perceptual learning effect, Artigas and Prados (2014) assessed the transfer of perceptual learning to novel compound stimuli sharing the $\mathrm{X}$ element in the absence of A and B. As previously described, they found, using a flavor aversion preparation that intermixed preexposure to $\mathrm{AX}$ and BX increased the discriminability of two novel compound stimuli, NX and ZX. Experiment 2 was designed to replicate this perceptual learning transfer using a standard appetitive Pavlovian procedure.

\section{Experiment 2}

Animals were preexposed to AX and BX according to an intermixed or a blocked schedule. Following preexposure, all animals were given conditioning trials with a new compound stimulus, NX, followed by a generalization test with yet another new compound, ZX. For this experiment, four stimuli were needed as the elements A, B, N and Z. The two sets of tones used in Experiment 1 were used: two tones of different frequency (Set 1) two discontinuous tones presented at different rates of intermittence (Set 2). Half of the subjects were preexposed to Set 1, and conditioned and tested with Set 2 . For the remaining animals, this arrangement was reversed. The actual stimuli that played the role of $\mathrm{A}$ and $\mathrm{B}$, or N and Z, were, therefore, fully counterbalanced across animals.

\section{Method}

\section{Subjects and apparatus}

The subjects were 16 experimentally naïve male hooded Long Evans rats (ad lib body weight range 240:393 g). The animals' maintenance and the apparatus were the same as that used in the previous experiment.

\section{Procedure}

The procedure of Experiment 2 replicates the procedural details described for Experiment 1. However, during the preexposure phase, the animals were preexposed to two of the four compound tones, $\mathrm{AX}$ and $\mathrm{BX}$, and were subsequently conditioned with a different compound (NX) and tested in the presence of a fourth compound (ZX). The four tones described for Experiment 1 were used in the way described above.

\section{Results and discussion}

Figure 3 shows the group means for the magazine approach response during the conditioning trials with the NX compound. An ANOVA with Preexposure (intermixed vs. blocked) and Blocks of Trials as factors showed a significant effect of Blocks of Trials, $F(3,42)=22.31, p=.00, \eta_{\mathrm{p}}^{2}=.61,95 \% \mathrm{CI}$ $[.38, .71]$. Neither the Preexposure factor nor the Preexposure $\times$ Blocks of Trials interaction was significant, $F_{\mathrm{S}}<1$.

The same analysis was carried out on the Pre-CS levels of responding during the conditioning phase. The mean Pre-CS scores for the four blocks of conditioning trials were 1.58 $( \pm 0.51$ SEM), $1.57( \pm 0.47), 1.65( \pm 0.44)$, and $1.95( \pm 0.55)$ for group intermixed; $1.45( \pm 0.41), 1.58( \pm 0.40), 1.66$ $( \pm 0.66)$, and $1.97( \pm 0.92)$ for group blocked. An ANOVA with

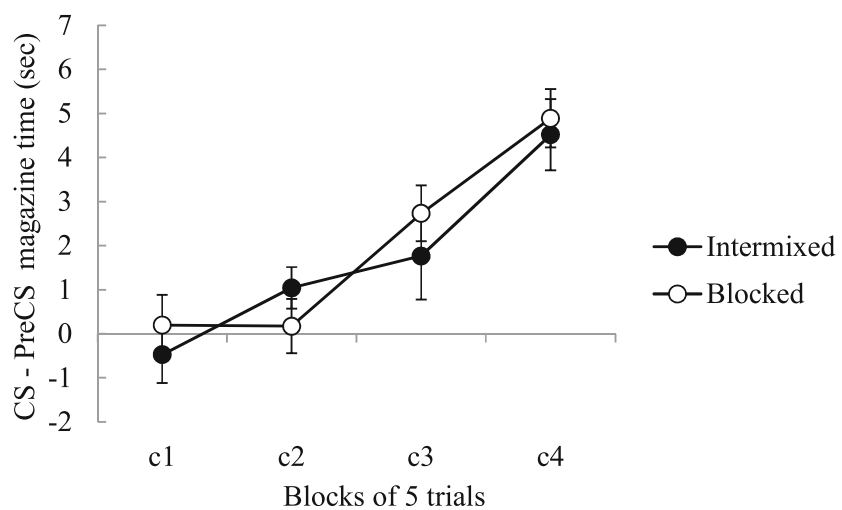

Fig. 3 Mean time $( \pm S E M)$ of magazine approach response calculated from difference scores (CS-Pre-CS) during the four blocks of conditioning trials with the compound $\mathrm{NX}$ for groups intermixed and blocked in Experiment 2 
Preexposure (intermixed vs. blocked) and Blocks of Trials as factors showed no significant effects, $F \mathrm{~s}<1$.

Figure 4 shows the group means for the magazine approach response during the test trials with the ZX compound. An ANOVA with Preexposure (intermixed vs. blocked) and Blocks of Test Trials as factors showed a significant effect of Preexposure, $F(1,14)=6.14, p=.02, \eta_{\mathrm{p}}{ }^{2}=.30,95 \%$ CI $[.00$, .56]. Neither the factor Blocks of Test Trials nor the interaction was significant, $F \mathrm{~s}<1$.

The same analysis was carried out on the Pre-CS scores during the test phase of the experiment. The mean Pre-CS scores for the three blocks of test trials were $1.27( \pm 0.62)$, $1.62( \pm 0.93)$, and $1.10( \pm 0.69)$ for group intermixed; 0.64 $( \pm 0.33), 1.65( \pm 0.84)$, and $0.77( \pm 0.75)$ for group blocked. An ANOVA with Preexposure (intermixed vs. blocked) and Blocks of Test Trials as factors showed no significant effects, $F_{\mathrm{s}}<1$.

Experiment 2 fully replicates the perceptual learning transfer effect observed by Artigas and Prados (2014) using a standard appetitive Pavlovian conditioning task instead of a flavor aversion discrimination task: Intermixed preexposure to AX and $\mathrm{BX}$ significantly reduced the generalization between two novel compound stimuli, NX and ZX. Given that $\mathrm{N}$ and $\mathrm{Z}$ are novel stimuli, we can assume that they would have a similar salience or effectiveness for the animals in the intermixed and blocked groups. One possibility that we need to discuss is the possible generalization of the effects of preexposure from A and $\mathrm{B}$ to the $\mathrm{N}$ and $\mathrm{Z}$ elements used during the generalization test of the experiment. Some perceptual learning theories (e.g., Hall, 2003) suggest that intermixed preexposure maintains high salience or effectiveness of A and B, whereas blocked preexposure reduces it. Transfer of low salience from $A B$ to

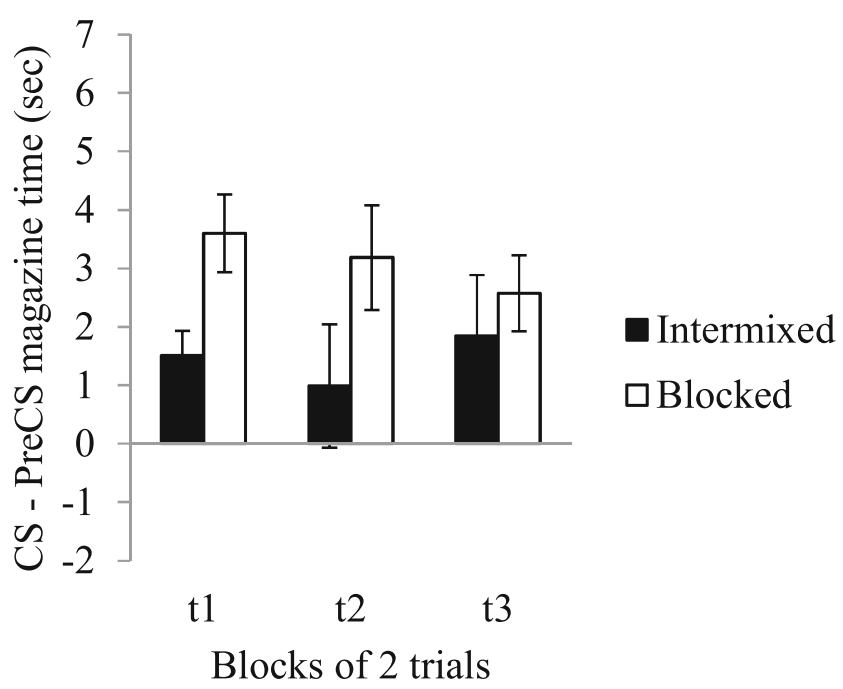

Fig. 4 Mean time $( \pm S E M)$ of magazine approach response calculated from difference scores (CS-Pre-CS) during the three blocks of test trials with the compound ZX for groups intermixed and blocked in Experiment 2
$\mathrm{NZ}$ would result in less interference with the acquisition and expression of predictive value by the common element $X$ in the blocked condition. This would be, in principle, consistent with the perceptual learning transfer observed in this experiment. However, the use of very distinctive stimuli such as $\mathrm{AB}$ and NZ (continuous and discontinuous tones) would have limited or prevented this generalization. Furthermore, Mondragon and Murphy (2010; see also Mondragon \& Hall, 2002) have shown that the $X$ element is certainly less salient in the intermixed than in the blocked condition. All in all, the perceptual learning transfer seems to be better explained in terms of differential perceptual effectiveness of the common element $X$ than as a result of differential generalization from $\mathrm{AB}$ to NZ. The reason the common element $\mathrm{X}$ is more susceptible to the effects of preexposure in the intermixed arrangement is the focus of the General Discussion.

\section{General discussion}

Our research was designed to address the mechanisms of perceptual learning assessed through generalization tests in a standard Pavlovian conditioning task. In spite of the fact that perceptual learning is supposed to depend upon associative learning and has been reported in different species using a variety of discrimination tasks and sensory modalities, its generality had not been extended to standard appetitive Pavlovian preparations until recently. Only one study, reported by Mondragon and Murphy (2010), had been able to demonstrate perceptual learning in an appetitive Pavlovian task. Experiment 1 replicated the basic effect reported by Mondragon and Murphy using the same stimuli they used in their experiments (tones of different frequencies; Experiment $1 \mathrm{~A}$ ) as well as with a new set of stimuli (discontinuous tones presented at different rates of intermittency; Experiment 1B). These two sets of stimuli were then used in Experiment 2 to explore whether the perceptual learning transfer effect observed by Artigas and Prados (2014) using a flavor discrimination task could also be observed using a more orthodox appetitive preparation. In Experiment 2, animals given intermixed preexposure to $\mathrm{AX}$ and $\mathrm{BX}$ showed improved discrimination of two novel compounds, NX and ZX, in comparison with animals given blocked preexposure. In the perceptual learning transfer test, $\mathrm{N}$ and $\mathrm{Z}$ are novel stimuli, identical for all the animals; therefore, the differences observed between the groups given intermixed and blocked preexposure should be attributed to the differential salience of the common element, $\mathrm{X}$.

Artigas and Prados (2014) suggested that the best way to account for the perceptual learning transfer, on the one hand, and the reduced conditioning of the $\mathrm{X}$ element observed by Mondragon and Murphy (2010; Mondragon \& Hall, 2002), on the other, is by reference to the different representations 
that might arise from intermixed and blocked preexposure to AX and BX: elemental in the case of intermixed and configural in the case of blocked preexposure. The hypothesis is based upon the principles of the stimulus sample theory (Atkinson \& Estes, 1963; Estes, 1959; McLaren \& Mackintosh, 2000), which presume stimuli to be formed by elements that are sampled in every presentation. During blocked preexposure to AX, for example, the elements of A and $\mathrm{X}$ can confidently be expected to be sampled at the same rate- $\mathrm{A}$ and $\mathrm{X}$ can be assumed to be equally salient. ${ }^{1}$ Coactivation of the elements of $\mathrm{A}$ and $\mathrm{X}$ in each preexposure trial would lead to the establishment of associations among them ( $x-a$ associations), as well as associations between the elements of $\mathrm{X}(x-x)$ and $\mathrm{A}(a-a)$. The existence of strong $x-a$ associations would contribute to the establishment of a unique configural-like representation of AX.

In the intermixed preexposure procedure, alternation of $A X$ and $\mathrm{BX}$ would result in a different sampling rate for the unique and the common elements: the $\mathrm{X}$ elements would be sampled in every trial, whereas the A (and B) elements would be sampled only every two trials. In the first AX trial, for example, we can expect the establishment of associations between the $\mathrm{X}$ elements $(x-x)$, the A elements $(a-a)$, and the $\mathrm{X}$ and $\mathrm{A}$ elements $(x-a)$; however, the $x-a$ associations can be expected to weaken in the absence of A during the subsequent BX trials, in which new links will be established between $x-x, b-b$ and $x-b$ (which will be weakened in the following AX trials). In the long term, we can expect the development of strong $x-x$, $a-a$, and $b-b$ associations, and relatively weak $x-a$ and $x-b$ associations. The final outcome of intermixed preexposure would therefore tend to be elemental representations of the elements $\mathrm{X}, \mathrm{A}$, and $\mathrm{B}$ rather than configural-like $\mathrm{AX}$ and BX representations (which would require strong $x-a$ and $x-$ $b$ associations).

All the representations that arise from intermixed and blocked preexposure would be subject to the effects of preexposure: loss of salience and associability. How this preexposure effects affect the conditioning of the $\mathrm{X}$ element presented by itself (as in the experiments by Mondragon \& Murphy, 2010) or in compound with a novel element (NX, as

\footnotetext{
${ }^{1}$ Our analysis develops the argument put forward by McLaren and Mackintosh (2000, p. 226) about the sampling process in the perceptual learning effect reported by Gibson and Walk (1956). In this study, rats showed enhanced discrimination learning between black geometrical figures (circle and triangle) that had been continuously exposed in the home cage of the animals. According to McLaren and Mackintosh, even when exposed to one such stimulus (rather than both of them simultaneously), the animals would be more likely to process the obvious common elements (black color) than the less salient distinctive geometric features (only evident from particular perspectives), leading to differential sampling of the common and the unique elements. In these experiments, using very different preexposure procedures, only intermixed preexposure would be likely to result in differential sampling; in the blocked condition, equal salience of $\mathrm{A}$ and $\mathrm{X}$ ( $\mathrm{a}$ white noise and a tone) would result in equivalent sampling of their elements.
}

in this experiment) can be expected to be different in the intermixed and blocked conditions. It is worth noting that in these experiments, the element $\mathrm{X}$ was presented in different contexts: in the presence of $\mathrm{A}$ and $\mathrm{B}$ during preexposure and in the presence of $\mathrm{N}$ and $\mathrm{Z}$ during discrimination training (our Experiment 2), or by itself (in the experiment by Mondragon \& Murphy, 2010). We can refer to the common element during preexposure, where it is always presented with A or B, as $X_{P}$, and during the conditioning or discrimination training, where it is presented either by itself or in the company of $\mathrm{N}$ or Z, as $X_{T}$. The effects of preexposure (habituation and latent inhibition) upon $X_{P}$ would generalize to $X_{T}$ only if they are perceived as similar stimuli by the individuals. It is reasonable to assume that there would be more similarity between the elemental representation $X_{P}$ and $X_{T}$ in the intermixed condition than between the configural representation $A X_{P}$ and $X_{T}$ in the blocked condition. As a result, high habituation and latent inhibition of $X_{T}$ in the intermixed condition would limit the capacity of the common element to enter into association with the US, reducing its conditioning (as observed by Mondragon \& Murphy, 2010), and limiting the generalization of conditioning from NX to ZX (as observed in Experiment 2).

As an alternative, Mondragon and Murphy (2010) suggested that differential changes in the salience of the X element in intermixed and blocked schedules of preexposure could be due to the influence of a selective attention mechanism "in the spirit" of that proposed by Mackintosh (1975). According to Mackintosh, stimuli perceived as good predictors of their outcomes would be preferentially attended, whereas poor predictors would be ignored. During blocked preexposure, $\mathrm{X}$ is paired with $\mathrm{A}$ for a number of trials (and then with B in a separate block of trials). In this way, X could be said to be a good predictor of the unique elements A and B, and its salience should increase. In the intermixed preexposure schedule, however, $\mathrm{X}$ is paired with $\mathrm{A}$ and $\mathrm{B}$ in alternating trials and would therefore be perceived as a poor predictor of the unique features $\mathrm{A}$ and $\mathrm{B}$; as a consequence, the salience of X could be expected to decline. Artigas and Prados (2014), however, pointed out that the opposite prediction could be made from other selective attention models. According to Pearce and Hall (1980), stimuli perceived as good predictors of their consequences would be ignored, whereas stimuli whose consequences are not certain would be preferentially attended. In the absence of a clear criterion to select one attentional mechanism or the other, selective attention models would remain ambiguous.

A different approach would be to assume that selective attention depends upon different attentional mechanisms that refer to different aspects of the stimulus. The hybrid model proposed by Le Pelley (2004) and Pearce and Mackintosh (2010) assumes the existence of two different parameters that modulate how a stimulus would be processed: attentional associability, which determines which stimuli would have 
access to the learning process, and salience associability, which controls the rate at which each stimulus will be learnt. The former would vary according to the principles put forward by Mackintosh (1975), and can be identified as $\alpha$; the latter would vary according to the principles advocated by Pearce and Hall (1980), and can be identified as $\sigma$. Le Pelley (2004) has argued for the convenience in a hybrid model that the value of $\alpha$ varies between 0.05 and 1 , whereas $\sigma$ should adopt values between 0.5 and 1 . The net associability of a stimulus would depend upon the interaction between these two parameters. In the blocked preexposure condition, where $\mathrm{X}$ is a good predictor of the unique elements $\mathrm{A}$ and $\mathrm{B}$, the attentional associability would be high $(\alpha \approx 1)$ whereas the salience associability would be relatively low $(\sigma \approx 0.5)$. The net associability of the $\mathrm{X}$ element would therefore be relatively high $(\alpha \cdot \sigma \approx 0.5)$. In the intermixed condition, however, where $\mathrm{X}$ is a poor predictor of $\mathrm{A}$ and $\mathrm{B}$, attentional salience would tend to be low $(\alpha \approx 0.05)$, whereas the salience associability would be high $(\sigma \approx 1)$. The net associability would therefore be lower than in the blocked condition $(\alpha \cdot \sigma \approx 0.05)$. This would account for the lower conditioning of $\mathrm{X}$ after intermixed than blocked preexposure in the experiments reported by Mondragon and Murphy (2010) as well as for the perceptual learning transfer reported in the present Experiment 2.

To conclude, this research adds to the previous report by Mondragon and Murphy (2010) in showing that the generality of the perceptual learning effect extends to standard appetitive Pavlovian tasks (Experiment 1). Furthermore, tit confirms that preexposure to $\mathrm{AX}$ and $\mathrm{BX}$ improves the discriminability of two novel compound stimuli, NX and ZX, a perceptual learning transfer effect (Experiment 2). A way to account for the differential effectiveness of the common element $\mathrm{X}$ after intermixed and blocked preexposure is by reference to the way in which the compound stimuli $\mathrm{AX}$ and $\mathrm{BX}$ are processed: elemental in the case of intermixed preexposure, and configural in the case of the blocked preexposure. An elemental representation of the $\mathrm{X}$ element after intermixed preexposure would facilitate the generalization of the effects of preexposure (latent inhibition, habituation) to the element $\mathrm{X}$ when it is encountered in a novel context during the discrimination phase of the experiment. This generalization can be expected to be lower between the configural cues established during blocked preexposure (AX and BX) and the $\mathrm{X}$ element encountered during the discrimination task. As an alternative, a hybrid selective attentional model (Le Pelley, 2004; Pearce \& Mackintosh, 2010) could provide a good explanation for the differences observed in the associability of $\mathrm{X}$ in the intermixed and blocked conditions.

Author note This work was supported by a grant (Ref: PSI201126850) from the Spanish Ministerio de Ciencia e Innovación to the authors.

\section{References}

Artigas, A. A., \& Prados, J. (2014). Perceptual learning transfer: Salience of the common element as a factor contributing to the intermixed/ blocked effect. Journal of Experimental Psychology: Animal Learning and Cognition, 40, 419-424.

Artigas, A. A., Sansa, J., Blair, C. A. J., Hall, G., \& Prados, J. (2006). Enhanced discrimination between flavor stimuli: Roles of salience modulation and inhibition. Journal of Experimental Psychology: Animal Behavior Processes, 32, 173-177.

Artigas, A. A., Sansa, J., \& Prados, J. (2006). The Espinet and the perceptual learning effects in flavour aversion conditioning: Do they depend on a common inhibitory mechanism? The Quarterly Journal of Experimental Psychology, 59, 471-481.

Atkinson, R. C., \& Estes, W. K. (1963). Stimulus sampling theory. In R. D. Luce, R. Bush, \& E. Galanter (Eds.), Handbook of mathematical psychology (Vol. 2, pp. 121-168). New York: Wiley.

Blair, C. A. J., \& Hall, G. (2003). Perceptual learning in flavor aversion: Evidence for learned changes in stimulus effectiveness. Journal of Experimental Psychology: Animal Behavior Processes, 29, 39-48.

Contel, D. M., Sansa, J., Artigas, A. A., \& Prados, J. (2011). Salience modulation and associative inhibition interaction: Short but not long exposure to similar stimuli protects the salience of the unique elements. Behavioural Processes, 86, 21-29.

Dwyer, D. M., Hodder, K. I., \& Honey, R. C. (2004). Perceptual learning in humans: Role of preexposure schedule, feedback and discrimination assay. The Quarterly Journal of Experimental Psychology, 57B, $245-259$.

Estes, W. K. (1959). The statistical approach to learning theory. In S. Koch (Ed.), Psychology, a study of science (Vol. 2, pp. 380-491). New York: McGraw-Hill.

Gibson, E. J. (1969). Principles of perceptual learning and development. New York: Appleton-Century-Crofts.

Gibson, E. J., \& Walk, R. D. (1956). The effect of prolonged exposure to visually presented patterns on learning to discriminate them. Journal of Comparative and Physiological Psychology, 49, 239-242.

Hall, G. (2003). Learned changes in the sensitivity of stimulus representations: Associative and nonassociative mechanisms. The Quarterly Journal of Experimental Psychology, 56B, 43-55.

Hall, G., Blair, C. A. J., \& Artigas, A. A. (2006). Associative activation of stimulus representations restores lost salience: Implications for perceptual learning. Journal of Experimental Psychology: Animal Behavior Processes, 32, 145-155.

Honey, R. C. (1990). Stimulus generalization as a function of stimulus novelty and familiarity in rats. Journal of Experimental Psychology: Animal Behavior Processes, 16, 178-184.

Honey, R. C., \& Bateson, P. (1996). Stimulus comparison and perceptual learning: Further evidence and evaluation from an imprinting procedure. The Quarterly Journal of Experimental Psychology, 49B, 259-269.

Honey, R. C., Bateson, P., \& Horn, G. (1994). The role of stimulus comparison in perceptual learning: An investigation with the domestic chick. The Quarterly Journal of Experimental Psychology, 47B, 83-103.

Lavis, Y., \& Mitchell, C. J. (2006). Effects of preexposure on stimulus discrimination: An investigation of the mechanisms responsible for human perceptual learning. The Quarterly Journal of Experimental Psychology, 59, 2083-2101.

Le Pelley, M. E. (2004). The role of associative history in models of associative learning: A selective review and a hybrid model. The Quarterly Journal of Experimental Psychology, 57B, 193-243.

Mackintosh, N. J. (1975). A theory of attention: Variations in the associability of stimuli with reinforcement. Psychological Review, 82, 276-298.

Mackintosh, N. J., Kaye, H., \& Bennett, C. H. (1991). Perceptual learning in flavour aversion learning. Quarterly Journal of Experimental Psychology, 43B, 297-322. 
McLaren, I. P. L., \& Mackintosh, N. J. (2000). An elemental model of associative learning: I. Latent inhibition and perceptual learning. Animal Learning \& Behavior, 28, 211-246.

Mitchell, C., \& Hall, G. (2014). Can theories of animal discrimination explain perceptual learning in humans? Psychological Bulletin, 140, 283-307.

Mitchell, C., Nash, S., \& Hall, G. (2008). The intermixed-blocked effect in human perceptual learning is not the consequence of trial spacing. Journal of Experimental Psychology: Learning, Memory, and Cognition, 34, 237-242.

Mondragon, E., \& Hall, G. (2002). Analysis of the perceptual learning effect in flavor aversion learning: Evidence for stimulus differentiation. The Quarterly Journal of Experimental Psychology, 55B, 153-169.

Mondragon, E., \& Murphy, R. A. (2010). Perceptual learning in an appetitive Pavlovian procedure: Analysis of the effectiveness of the common element. Behavioural Processes, 83, 247-256.

Mundy, M. E., Honey, R. C., \& Dwyer, D. M. (2007). Simultaneous presentation of similar stimuli produces perceptual learning in human picture processing. Journal of Experimental Psychology: Animal Behavior Processes, 33, 124-138.

Pearce, J. M., \& Hall, G. (1980). A model for Pavlovian conditioning: Variations in the effectiveness of conditioned but not of unconditioned stimuli. Psychological Review, 87, 532-552.
Pearce, J. M., \& Mackintosh, N. J. (2010). Two theories of attention: A review and a possible integration. In C. J. Mitchell \& M. E. Le Pelley (Eds.), Attention and associative learning: From brain to behaviour. Oxford: Oxford University Press.

Prados, J., Artigas, A. A., \& Sansa, J. (2007). Preexposure effects in the spatial domain: Dissociation between latent inhibition and perceptual learning. Journal of Experimental Psychology: Animal Behavior Processes, 33, 115-123.

Prados, J., Chamizo, V. D., \& Mackintosh, N. J. (1999). Latent inhibition and perceptual learning in a swimming-pool navigation task. Journal of Experimental Psychology: Animal Behavior Processes, 25, 37-44.

Prados, J., Hall, G., \& Leonard, S. (2004). Dissociation between the Espinet and perceptual learning effects in flavour aversion conditioning. Behavioural Processes, 65, 221-229.

Rescorla, R. A., \& Wagner, A. R. (1972). A theory of Pavlovian conditioning: Variations in the effectiveness of reinforcement and nonreinforcement. In A. H. Black \& W. F. Prokasy (Eds.), Classical conditioning II: Current research and theory (pp. 6499). New York: Appleton-Century-Crofts.

Steiger, J. H. (2004). Beyond the $F$ test: Effect size confidence intervals and tests of close fit in the analysis of variance and contrast analysis. Psychological Methods, 9, 164-182. 